\title{
Finding and Fixing Bugs in Web Applications (Invited Talk)
}

\author{
Frank Tip \\ IBM Thomas J. Watson Research Center \\ Hawthorne, NY USA \\ ftip@us.ibm.com
}

\begin{abstract}
Today's society is critically dependent on the existence of web applications. From online purchases to personal banking to mobile devices, web applications are the backbone of the 21st century's economy. However, web applications have a number of characteristics that make them highly fragile and prone to bugs that threaten the important applications they enable. In particular, they are typically written in a combination of multiple languages, they often rely on low-level manipulation of string values to generate dynamic web page content, and the flow of control in web applications usually depends strongly on interactive input from the user. In this presentation, I will present an overview of the Apollo project at IBM Research, which aims to make web applications more robust by assisting programmers with finding and fixing bugs, using automated techniques for test generation, fault localization, and program repair.
\end{abstract}

